\title{
Compreendendo as propriedades (estrutural, espectroscópica, colorimétrica e termica) de sais de níquel
}

\section{Understanding the properties (structural, spectroscopic, colorimetric and thermal) of nickel salts}

Tamara Maria de Andrade ${ }^{1}$, Felipe Quadros Mariani ${ }^{1}$, Cícero Venâncio Nunes Júnior ${ }^{1}$, Mariane Dalpasquale ${ }^{1}$, Marins Danczuk ${ }^{1}$, Fauze Jacó Anaissi ${ }^{1}$

\footnotetext{
${ }^{1}$ Laboratório de Materiais - LabMat - UNICENTRO, Rua Simeão Varela de Sá 03, CEP: 85040-080, Guarapuava, PR e-mail: andrade.tamaraa@gmail.com,fqmariani@gmail.com, cicero_labmat@gmail.com, ane_dalpasquale@hotmail.com, marinsdk@yahoo.com.br; fjanaissi@gmail.com
}

\section{RESUMO}

Sais de níquel (acetato, cloreto e nitrato) são de uso corriqueiro nos laboratórios de síntese inorgânica e orgânica. No processo de obtenção de óxidos, hidróxidos e oxi-hidróxidos de níquel para as mais diversas aplicações (baterias, pigmentos, sensores e eletrocatálise, por exemplo) se faz necessário compreender as características, as propriedades estruturais e eletrônicas, que cada sal precursor pode conferir ao produto final. Como premissa, foi realizado uma avaliação do comportamento estrutural, espectroscópico e térmico de sais de níquel. O perfil de difração de raios X (DRX) possibilitou identificar a rede cristalina e seus parâmetros. Dados espectroscópicos (UV-Vis) mostram a similaridade de absorção em solução e as bandas atribuídas de acordo com as transições permitidas e proibidas para o íon hexa(aquo)níquel(II). Porém, os espectros se diferenciam quando se avalia os sólidos por refletância difusa. Fato que possibilita calcular as energias de band gap para cada sal; acetato $2,85 \mathrm{eV}$, cloreto $2,46 \mathrm{eV}$ e nitrato 2,61 eV. Dados colorimétricos ( $\mathrm{L}^{*} \mathrm{a} * \mathrm{~b}$ ) permitem prever que a intensidade de tom verde diminui na sequência: nitrato > cloreto > acetato. Curvas térmicas (TG/DTA) inferem grau de pureza e quantificam o número de moléculas de água de hidratação, compatíveis com os dados do fornecedor. A análise morfológica (MEV) e estrutural (DRX) das partículas geradas nas curvas térmicas permitiram determinar a composição estimada de $\mathrm{NiO}$ residual, com maior precisão para o derivado do nitrato de níquel $\left(\mathrm{NiO}_{1,1}\right)$, com tamanho de partícula da ordem de $0,1 \mu \mathrm{m}$, o menor entre os resíduos.

Palavras-chave: Sais de níquel, caracterização, espectroscopia, colorimetria.

\begin{abstract}
Nickel salts (acetate, chloride and nitrate) are of everyday use in laboratories of inorganic and organic syntheses. In the process of obtaining oxides, hydroxides and oxy-hydroxides of nickel for the most diverse applications (batteries, pigments, sensors and electrocatalysis for example) it is necessary to understand the characteristics, the structural and electronic properties, that each precursor salt can confer to the final product. Premised carry out an assessment of the structural, spectroscopic and thermal behavior of nickel salts. Profile $\mathrm{X}$-ray diffraction (XRD) enabled the identification of the crystal lattice and its parameters. Spectroscopic data (UV-Vis) show the similarity of solution absorption bands assigned in accordance with the permitted and prohibited transitions to hexa(aqua)nickel(II) ion. However, the spectra differ when evaluating the solids by diffuse reflectance. Fact that enables to calculate the band gap energies for each salt; value of $2.85 \mathrm{eV}$ for acetate, $2.46 \mathrm{eV}$ for chloride and $2.61 \mathrm{eV}$ for nitrate. Color data $\left(\mathrm{L}^{*} \mathrm{a} * \mathrm{~b} *\right)$ can predict the green ton of intensity decreases in the sequence: nitrate> chloride> acetate. Curves thermal (TG-DTA) infer purity and quantify the number of hydration water molecules, consistent with the supplier information. Morphological analysis (SEM) and structural (XRD) of the particles generated in the thermal curves allow to determine the estimated residual $\mathrm{NiO}$ composition, more precisely to the derivative of nickel nitrate $\left(\mathrm{NiO}_{1.1}\right)$ whose particle size is of the order of $0.1 \mu \mathrm{m}$, the smaller size from the waste.
\end{abstract}

Keywords: Nickel salts; Characterization; Spectroscopy, Colorimetry. 


\section{INTRODUÇÃO}

A química de materiais relaciona de forma direta a visão atômica/molecular com as propriedades específicas para o desenvolvimento de determinadas funções. E como afirma ZARBIN [1] "toda matéria é um material em potencial", e o que vai determinar a capacidade dessa matéria em atuar como material são as características e as propriedades que proporcionam funções específicas de um material.

O processo para o desenvolvimento de novos materiais vem se renovando a cada século. Isso porque desde a idade da pedra, do bronze e do ferro, o homem tem utilizado o que tem a seu dispor para as mais diversas finalidades, e a cada momento ele fez, e ainda faz, as devidas adaptações para que os materiais sejam aperfeiçoados e contribuam para o desenvolvimento social, cultural entre outros. Os "novos" materiais são obtidos através de um processo básico constituído por quatro etapas: aplicação, propriedades, estrutura e rota de síntese. Para aplicações específicas de um determinado material é preciso saber quais as propriedades necessárias para tal aplicação e, com isso, entender qual a estrutura e a composição do material capaz de conferir as propriedades desejadas e, por fim, definir qual a melhor rota de síntese capaz de produzir o material com as propriedades e estrutura necessária para a aplicação de interesse.

Inserido nesse contexto, se encontram diversos materiais a base de hidróxido de níquel e óxido de níquel, entre outros. A presença deste metal na crosta terrestre é de aproximadamente $0,01 \%$, sendo encontrado sob a forma de sulfito, óxidos e silicato mineral. Sua distribuição está em níveis moderados em função de ações naturais, como intemperismo e atividades vulcânicas [2]. A sua importância comercial é extremamente grande, em função da vasta possibilidade de aplicações, como por exemplo, na síntese de óxidos [3-4], hidróxidos e oxi-hidróxidos[5-10] e derivados de sais inorgânicos de níquel ou ainda, em aplicações como baterias [11-13], sensores amperométricos [14-16], eletrocatálise [17,18], dispositivos eletrocrômicos [19] e supercapacitores [20].

Dentre os diversos sais inorgânicos de níquel, o acetato, cloreto e nitrato de níquel, são amplamente utilizados como fonte de íons $\mathrm{Ni}(\mathrm{II})$ [5] por serem solúveis em água, baixo custo e de fácil manipulação, pois não requerem condições especiais de trabalho (glove box, atmosfera inerte, etc.), tornando-os importantes em metodologias que visam preparar materiais eletroativos que atendam aplicações múltiplas [5-7]. Sais de níquel são objetos de estudo na síntese de hidróxido de níquel. Em 2015, NUNES et al. [21] avaliaram a síntese de $\alpha-\mathrm{Ni}(\mathrm{OH})_{2}$ a partir do sal acetato de níquel com $\mathrm{NaOH}$, utilizando a argila bentonita como hóspede, obtendo maior organização estrutural e alta densidade de corrente quando os materiais passam por processo de liofilização.

Tendo em vista o crescente estudo de síntese de hidróxido de níquel (fases mistas ou puras), este trabalho tem como objetivo expandir e reunir informações disponíveis acerca dos sais de níquel (acetato, cloreto e nitrato) amplamente utilizados nesses estudos. A metodologia utilizada baseou-se na coleta experimental dos dados e apresentação do estudo comparativo e sistemático dos sais de níquel selecionados, por meio das técnicas de difratometria de raios X (DRX), espectroscopia eletrônica (UV-Vis), análise térmica (TG-DTGDTA) e microscopia eletrônica de varredura (MEV-EDS) e com isso contribuir durante o processo de escolha do sal precursor em um processo de síntese de materiais.

\section{MATERIAIS E MÉTODOS}

Três sais de níquel comerciais foram utilizados sem qualquer tratamento prévio de purificação ou de secagem (tratamento térmico). São eles: acetato de níquel tetra-hidratado $\left[\mathrm{Ni}^{(}\left(\mathrm{CH}_{3} \mathrm{COO}\right)_{2} \cdot 4 \mathrm{H}_{2} \mathrm{O}\right.$, grau P.A., PróQuímica], cloreto de níquel hexa-hidratado $\left[\mathrm{NiCl}_{2} \cdot 6 \mathrm{H}_{2} \mathrm{O}\right.$, grau P.A., Merck] e nitrato de níquel hexahidratado $\left[\mathrm{Ni}\left(\mathrm{NO}_{3}\right)_{2} \cdot 6 \mathrm{H}_{2} \mathrm{O}\right.$, grau P.A., Vetec]. Soluções aquosas foram preparadas utilizando água de grau ultrapura (18 M $\Omega . c m$ ) obtida de um sistema de osmose reversa da Quimis, modelo Q842 10L.

\subsection{Técnicas de Caracterização}

Com o objetivo de definir o grau de cristalinidade e identificar a fase formada foi realizada a análise estrutural por difração de raios X (DRX) dos sais. Os dados foram coletados em um difratômetro D2 Phaser da Bruker, com cátodo de cobre com emissão $\alpha \alpha(\lambda=1,5418 \AA)$, potência de $30 \mathrm{kV}$, corrente de $10 \mathrm{~mA}$ e varredura de $0.05^{\circ} 2 \theta / \mathrm{min}$, na região $2 \theta$ de $5^{\circ}$ a $80^{\circ}$. Os dados foram tratados no DIFFRAC.EVA, versão Bruker, software universal para avaliação de dados de difração de raios X integrado ao Powder Diffraction File (PDF-2 2009) para indexação das fases cristalinas a partir das fichas catalográficas do ICDD (International Centre for Diffraction Data).

Os espectros obtidos por transmissão (região do UV-Vis) foram coletados no espectrofotômetro Varian, modelo 3000 com o objetivo de definir as regiões de absorção e identificar as transições eletrônicas das 
amostras. Foram utilizadas soluções aquosas dos sais de níquel na concentração de $0,05 \mathrm{~mol} \mathrm{~L}^{-1}$, em cubeta de quartzo com $1 \mathrm{~cm}$ de caminho óptico. Os espectros eletrônicos para os sais na forma sólida foram obtidos em modo reflectância, em um espectrofotômetro Ocean Optics, modelo USB2000, acoplado a uma lâmpada de tungstênio (região de 380 - $1100 \mathrm{~nm}$ ) e um conjunto de fibra óptica dupla (lâmpada/amostra - amostra/detector) apropriado para amostras sólidas. Os espectros foram coletados no Spectra Suite, Spectrometer Operating Software, e os gráficos apresentados conforme obtido no modo espectro visível (Visible Spectrum) com espectro de cores, no intuito de explicitar as bandas de reflectância dos sólidos. O espectrofotômetro USB2000 proporcionou a análises de medidas de colorimetria pelo método CIELab, sistema utilizado para descrever matematicamente as percepções de cores $\left(\mathrm{L}^{*} \mathrm{a} * \mathrm{~b} *\right.$ ).

Para definir a estabilidade térmica e verificar o grau de hidratação dos sais, medidas de análise térmica foram realizadas em um analisador térmico simultâneo (TG-DTG-DTA) SII Nano Technology Inc., modelo TG/DTA 6300 com de coleta de dados TA Rheo System Software (MUSE Program). Nas condições de aquecimento na rampa de $15^{\circ} \mathrm{C} \mathrm{min}^{-1}$, atmosfera dinâmica de ar comprimido (vazão $200 \mathrm{~mL} \mathrm{~min}^{-1}$ ), em porta amostra (cadinho) de alumina, $12,39 \mathrm{mg}$ de $\mathrm{Ni}\left(\mathrm{CH}_{3} \mathrm{COO}\right)_{2} \cdot 4 \mathrm{H}_{2} \mathrm{O} ; 19,33 \mathrm{mg}$ de $\mathrm{NiCl}_{2} \cdot 6 \mathrm{H}_{2} \mathrm{O}$ e $4,75 \mathrm{mg}$ de $\mathrm{Ni}\left(\mathrm{NO}_{3}\right)_{2} \cdot 6 \mathrm{H}_{2} \mathrm{O}$. Os experimentos foram realizados entre $30{ }^{\circ} \mathrm{C}$ e $1200{ }^{\circ} \mathrm{C}$, e os dados analisados MUSE Standard Analysis. Os resíduos, ou seja, a massa final após o ensaio da análise térmica, foram utilizados para obter imagens de microscopia eletrônica de varredura (MEV) em um microscópio Hitachi TM-3000, com filamento de tungstênio como fonte de elétrons e potência de $10 \mathrm{KV}$. O MEV está acoplado ao detector SwiftED-3000, para análise elementar por energia dispersiva (EDS). As imagens foram analisadas no software livre Image (v. 1.4.1) e os dados utilizados para gerar os histogramas de distribuição de tamanho de partículas.

\section{RESULTADOS E DISCUSSÕES}

Neste trabalho está sendo apresentado um estudo comparativo do comportamento estrutural, espectroscópico e térmico dos sais de níquel com o propósito de facilitar discussões futuras e apresentar aos pesquisadores uma alternativa de avaliação de sais inorgânicos empregados corriqueiramente sem nenhum tratamento prévio de purificação.

\subsection{Comportamento Estrutural (DRX)}

A Figura 1 mostra o padrão de difração de raios $\mathrm{X}$ dos sais de níquel que foram indexados de acordo com as cartas cristalográficas: PDF 00-025-0901 para acetato de níquel com 79,3\% de cristalinidade; PDF 00-0251044 para cloreto de níquel com 77,4 \% de cristalinidade; e PDF 00-025-0577 para nitrato de níquel com $59,9 \%$ de cristalinidade.

Por se tratar de sais hidratados o grau de cristalinidade é considerado excelente, visto que sólidos hidratados sofrem alargamento nos picos de difração e geralmente são submetidos a tratamento térmico. Porém o objetivo da coleta dos dados de DRX para sais inorgânicos é identificar a rede cristalina e os parâmetros de rede, assim como os picos de difração característico de cada fase. O tipo de rede cristalina e os respectivos parâmetros associados aos picos de difração [2Ө/d( $\AA$ )/hkl] característico de cada sal de níquel, são destacados a seguir.

- Acetato de níquel tetra-hidratado, apresenta rede monoclínica (P21/a) e parâmetros de rede: $\mathbf{a}=$ 8,$44700 ; \mathbf{b}=11,77800 ; \mathbf{c}=4,77800 ; \mathbf{a} / \mathbf{b}=0,71718$ e $\mathbf{c} / \mathbf{b}=0,40567$. Picos de difração em: $\left[12,87^{\circ} / 6,87 / 110\right.$ $(100 \%)] ; \quad\left[18,58^{\circ} / 4,77 / 001\right]$; [21,03\%/4,22/200]; [24,97\%/3,56/130]; [28,30\%/3,15/-211]; [31,11\%/2,87/230]; [35,83\%/2,50/041]; [39,06\%/2,30/330] e [64,93\%/1,44/432].

- Cloreto de níquel hexa-hidratado, apresenta rede monoclínica $(\mathrm{C} 2 / \mathrm{m})$ e parâmetros de rede: $\mathbf{a}=$ 8,78600; $\mathbf{b}=7,07600 ; \mathbf{c}=6,62500 ; \mathbf{a} / \mathbf{b}=1,24166$ e $\mathbf{c} / \mathbf{b}=0,93626$. Picos de difração em: [15,84\% $/ 5,59 /-101]$; [16,10\%/5,50/110]; [18,39\%/4,82/011 (100\%)]; [25,12\%/3,54/020]; [30,52\%/2,92/-112]; [33,29\%/2,68/310]; $\left[35,26^{\circ} / 2,54 / 301\right] ; \quad\left[37,32^{\circ} / 2,40 / 022\right] ; \quad\left[41,42^{\circ} / 2,17 / 400\right] ; \quad\left[46,01^{\circ} / 1,97 / 312\right] ; \quad\left[48,87^{\circ} / 1,86 /-123\right] \quad$ e [59,68\%1,54/332].

- Nitrato de níquel hexa-hidratado, apresenta rede triclínica (P-1) e parâmetros de rede: a = 7,69900; $\mathbf{b}=11,67700 ; \mathbf{c}=5,79900 ; \mathbf{a} / \mathbf{b}=0,65933$ e $\mathbf{c} / \mathbf{b}=0,49662$. Picos de difração em: $\left[8,08^{\circ} / 10,93 / 010\right] ;$ $\left[16,16^{\circ} / 5,48 / 020\right] ; \quad\left[23,63^{\circ} / 3,76 /-210\right] ; \quad\left[26,08^{\circ} / 3,41 / 0-31\right] ; \quad\left[28,92^{\circ} / 3,08 /-230\right] ; \quad\left[31,05^{\circ} / 2,88 /-140\right]$; [32,64\% $/ 2,74 / 040(100 \%)]$; [39,45\%/2,28/-2-22] e [53,41\%/1,71/0-43].

Essas características conjuntas auxiliam na interpretação das fases presentes em novos materiais obtidos com os precursores de níquel (acetato, cloreto e nitrato), como por exemplo, excesso de reagente ou fração que não reagiu durante o preparo. 


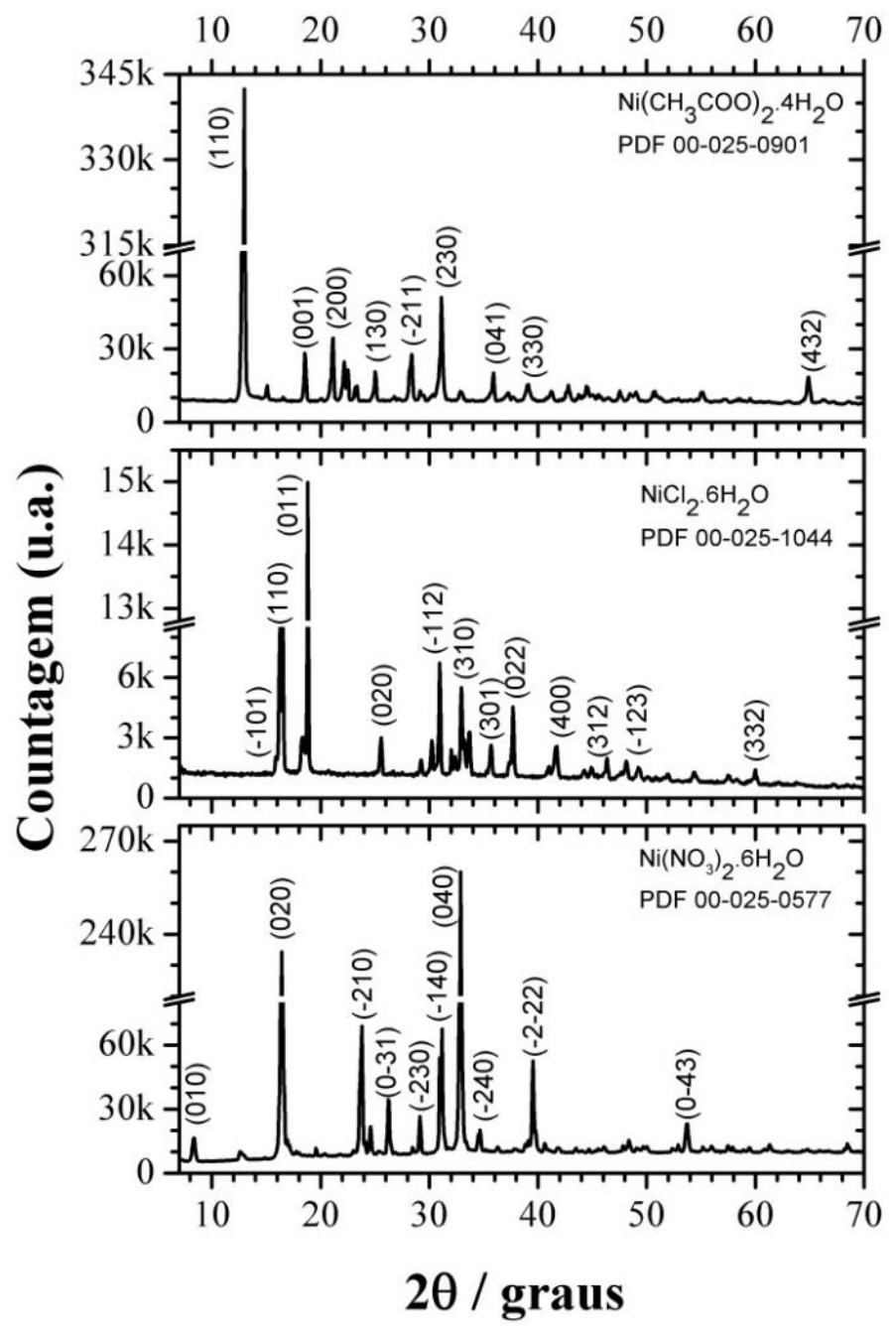

Figura 1: Padrão de difração de raios $X$ para os sais de níquel indexados de acordo com suas respectivas cartas cristalográficas PDF (Powder Diffraction File).

\subsection{Comportamento Espectroscópico (UV-Vis)}

Espectros eletrônicos são de uso constante para interpretar comportamento óptico de sais inorgânicos e compostos de coordenação envolvendo metais de transição. Seja na forma de soluções coloridas, na forma de pó ou pastilha [22], o espectro eletrônico pode ser obtido nos modos absorbância, transmitância, refletância ou irradiação (contagens por minuto). Na Figura 2A está apresentado o perfil espectral em solução dos sais de níquel em estudo, e na Figura 2B, o Diagrama de Tanabe-Sugano usado para a atribuição das transições eletrônicas apresentadas.

Na Figura 2A estão evidenciadas quatro regiões de transições d-d, sendo: duas permitidas por spin, ou seja, aquelas que ocorrem entre o estado fundamental e os estados excitados que apresentem mesma multiplicidade [23][24], $\left[{ }^{3} \mathrm{~A}_{2 \mathrm{~g}} \rightarrow{ }^{3} \mathrm{~T}_{1 \mathrm{~g}}(\mathrm{P})\right.$ em $\left.394 \mathrm{~nm}\right]$ e $\left[{ }^{3} \mathrm{~A}_{2 \mathrm{~g}} \rightarrow{ }^{3} \mathrm{~T}_{1 \mathrm{~g}}(\mathrm{~F})\right.$, em $735 \mathrm{~nm}$, e duas proibidas por Laporte (que não apresenta mudança de paridade), $\left[{ }^{3} \mathrm{~A}_{2 \mathrm{~g}} \rightarrow{ }^{1} \mathrm{~T}_{2 \mathrm{~g}}\right.$, em 500] e por spin $\left[{ }^{3} \mathrm{~A}_{2 \mathrm{~g}} \rightarrow{ }^{1} \mathrm{E}_{\mathrm{g}}\right.$, em $660 \mathrm{~nm}$ [23] função da limitação do espectrofotômetro utilizado $(200-800 \mathrm{~nm})$, a transição permitida por spin $\left[{ }^{3} \mathrm{~A}_{2 \mathrm{~g}} \rightarrow{ }^{3} \mathrm{~T}_{2 \mathrm{~g}}\right.$ (F)] que ocorre na região acima de $950 \mathrm{~nm}$, não foi observada [23].Considerando essa banda, os espectros eletrônicos de sais de níquel podem apresentar até cinco regiões de transições eletrônicas, sendo: três permitidas (caracterizadas por serem mais intensas) e duas proibidas.

Os espectros eletrônicos de sais de níquel (Figura 2) apresentam o mesmo perfil de absorção relativo às transições (d-d) atribuídas. Este comportamento é devido ao fato de que em solução aquosa, sais de $\mathrm{Ni}^{2+}$, sem a presença de agentes complexantes (ligantes $\pi$ receptores, por exemplo), ocorre predominantemente a formação do íon $\left[\mathrm{Ni}\left(\mathrm{H}_{2} \mathrm{O}\right)_{6}\right]^{2+}$, hexa(aquo)níquel(II), de coloração verde [25].

Na Tabela 1 estão sumarizadas as transições eletrônicas e suas atribuições de acordo com a literatura [23-25], e observado experimentalmente em solução (absorbância) e na forma de pó (refletância). 

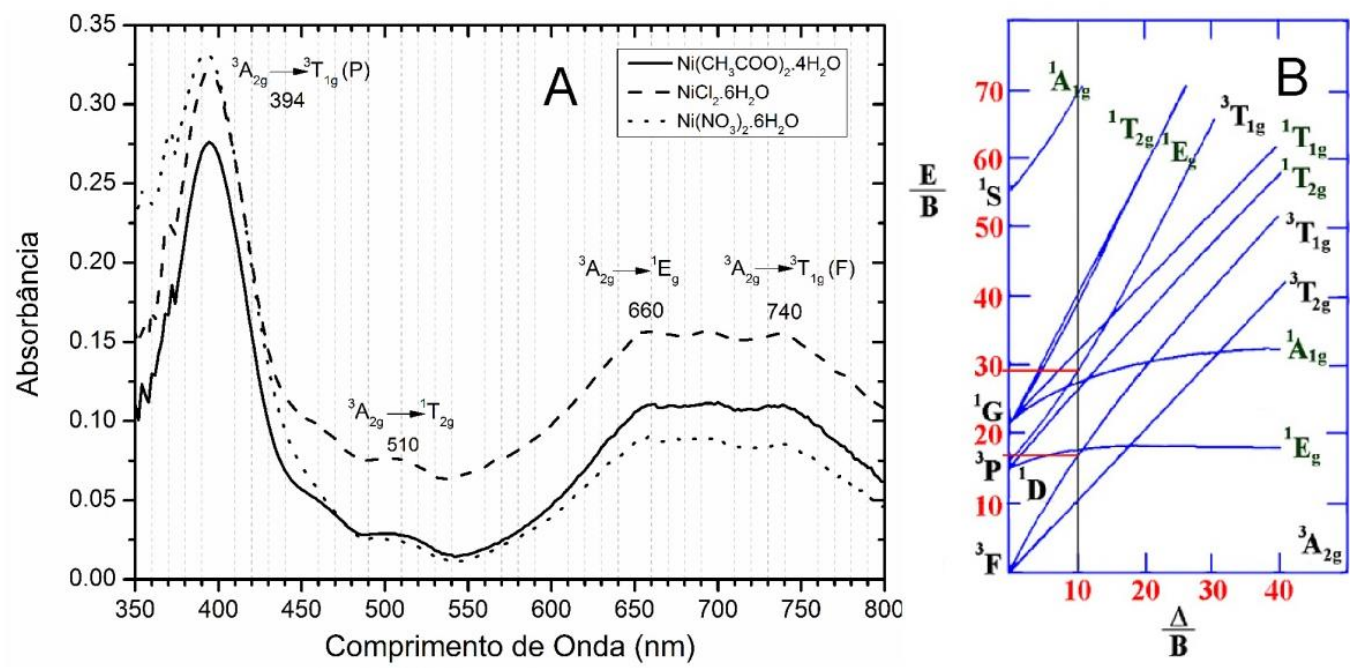

Figura 2: (A) Espectros eletrônicos (UV-Visível) das soluções aquosas de sais de níquel; (B) Diagrama de níveis de energia Tanabe-Sugano para íon $\mathrm{d}^{8}$ (adaptado ref. [23]).

\subsubsection{Calculando Parâmetros de Racah (B) e as energias de transições}

As atribuições dos espectros eletrônicos (Tabela 1) para os sais de níquel são típicas de geometria octaédrica, pois às transições observadas estão de acordo com as esperadas para o íon $\mathrm{d}^{8}$ em campo octaédrico. Deste modo, a partir do diagrama de Tanabe-Sugano (Figura 2B) é possível determinar os parâmetros devido ao desdobramento de campo cristalino (10 Dq) e a repulsão intereletrônica dos íons ou parâmetro de Racah (B) [26-27]. A partir dos valores de comprimento de onda de absorção máximo $\left(\lambda_{\max }\right)$ das transições (Figura $2 \mathrm{~A}-$ linha sólida), calcula-se os valores de energia $(E)$ para cada transição, partindo da conversão $v\left(\mathrm{~cm}^{-1}\right)=10^{7} / \lambda$ $(\mathrm{nm})$, tem-se:

- A primeira energia envolve a transição $\left[{ }^{3} \mathrm{~A}_{2 \mathrm{~g}} \rightarrow^{3} \mathrm{~T}_{1 \mathrm{~g}}(\mathrm{P})\right]$ :

$$
E_{1}=10^{7} / 394 \mathrm{~nm}=25.380,71 \mathrm{~cm}^{-1} \text {; }
$$

- A segunda energia corresponde a transição $\left[{ }^{3} \mathrm{~A}_{2 \mathrm{~g}} \rightarrow{ }^{3} \mathrm{~T}_{1 \mathrm{~g}}(\mathrm{~F})\right]$ :

$$
E_{2}=10^{7} / 735 \mathrm{~nm}=13.513,51 \mathrm{~cm}^{-1} .
$$

A razão entre as energias $\left(E_{1} / E_{2}\right)$ corresponde a 1,87 e assim, encontra-se o valor de $\Delta / \mathrm{B}=9 ; E_{1} / \mathrm{B}=$ 28 e $E_{2} / \mathrm{B}=15$ do diagrama de Tanabe-Sugano para íons d $\mathrm{d}^{8}$ (Figura 2B). Partindo desses valores então, o parâmetro de Racah é calculado para cada energia:

$$
\begin{aligned}
& B_{1}=25.380,71 / 28=906,45 \mathrm{~cm}^{-1} ; \\
& B_{2}=13.513,51 / 15=900,90 \mathrm{~cm}^{-1} ;
\end{aligned}
$$

$$
\rightarrow B_{\text {médio }}=903,67 \mathrm{~cm}^{-1}
$$

Para calcular o valor de $10 \mathrm{Dq}$, a partir da razão de $10 \mathrm{Dq}$ / $B$, sendo $B_{\text {médio }}$ o parâmetro de Racah: $\mathrm{Dq}=$ $\left(9,0 \times 903,67 \mathrm{~cm}^{-1}\right)=8133,00 \mathrm{~cm}^{-1}$. Os valores do parâmetro de Racah $\left(B=903,67 \mathrm{~cm}^{-1}\right)$ e de $10 \mathrm{Dq}(8133,00$ $\mathrm{cm}^{-1}$ ) determinados experimentalmente estão de acordo com os valores reportados na literatura $[23,28]$. 
Tabela 1: Transições eletrônicas para sais de níquel: em solução aquosa (Figura 2), na forma de pó (Figura 3) e reportadas na literatura $[23,29]$

\begin{tabular}{|c|c|c|c|c|c|c|}
\hline \multirow{2}{*}{ TRANSIÇÃO $(\mathrm{d}-\mathrm{d})^{8}$} & \multirow{2}{*}{$\begin{array}{c}\Delta_{\text {aquoso }} / \mathbf{n m} \\
\left(\mathbf{c m}^{-1}\right)\end{array}$} & \multicolumn{3}{|c|}{$\Delta_{\text {sólido }} / \mathrm{nm}$} & \multirow{2}{*}{$\begin{array}{l}\text { REGIÃOO } \\
(\mathbf{n m})^{*}\end{array}$} & \multirow{2}{*}{$\begin{array}{l}\text { LITERATURA } \\
(\mathbf{n m})\end{array}$} \\
\hline & & ACETATO & CLORETO & NITRATO & & \\
\hline $\begin{array}{l}{ }^{3} \mathrm{~A}_{2 \mathrm{~g}} \rightarrow{ }^{3} \mathrm{~T}_{1 \mathrm{~g}}(\mathrm{P}) \\
\quad \text { (permitida por spin) }\end{array}$ & $\begin{array}{c}394 \\
(25.380)\end{array}$ & --- & --- & --- & $364-423$ & 394 [23] \\
\hline $\begin{array}{l}{ }^{3} \mathrm{~A}_{2 \mathrm{~g}} \rightarrow{ }^{1} \mathrm{~T}_{2 \mathrm{~g}} \\
\quad \text { (proibida por Laporte) }\end{array}$ & $\begin{array}{c}510 \\
(19.600)\end{array}$ & 430 & 515 & 450 & $450-550$ & 457 [23] \\
\hline $\begin{array}{l}{ }^{3} \mathrm{~A}_{2 \mathrm{~g}} \rightarrow{ }^{1} \mathrm{E}_{\mathrm{g}} \\
\quad \text { (proibida por spin) }\end{array}$ & $\begin{array}{c}660 \\
(15.151)\end{array}$ & 590 & 650 & 580 & $625-690$ & 665 [23] \\
\hline $\begin{array}{l}{ }^{3} \mathrm{~A}_{2 \mathrm{~g}} \rightarrow{ }^{3} \mathrm{~T}_{1 \mathrm{~g}}(\mathrm{~F}) \\
\quad \text { (permitida por spin) }\end{array}$ & $\begin{array}{c}740 \\
(13.513)\end{array}$ & --- & --- & --- & $710-730$ & 724 [23] \\
\hline $\begin{aligned} &{ }^{3} \mathrm{~A}_{2 \mathrm{~g}} \rightarrow{ }^{3} \mathrm{~T}_{2 \mathrm{~g}}(\mathrm{~F}) \\
& \quad \text { (permitida por spin) }\end{aligned}$ & --- & --- & --- & --- & $977-999$ & 1200 [23] \\
\hline $\begin{array}{l}{ }^{3} \mathrm{~A}_{2 \mathrm{~g}} \rightarrow{ }^{3} \mathrm{~T}_{1 \mathrm{~g}}(\mathrm{P}) \\
\quad \text { (permitida por spin) }\end{array}$ & $\begin{array}{c}394 \\
(25.380)\end{array}$ & --- & --- & --- & $364-423$ & 394 [23] \\
\hline $\begin{array}{l}{ }^{3} \mathrm{~A}_{2 \mathrm{~g}} \rightarrow{ }^{1} \mathrm{~T}_{2 \mathrm{~g}} \\
\quad \text { (proibida por Laporte) }\end{array}$ & $\begin{array}{c}510 \\
(19.600)\end{array}$ & 430 & 515 & 450 & $450-550$ & 457 [29] \\
\hline
\end{tabular}

Obs: Região de absorção [26].

\subsubsection{Discutindo espectros no estado sólido e calculando energia de "band gap" óptico}

Na Figura 3A são mostrados os espectros eletrônicos por refletância difusa dos sais de níquel na forma de pó (sólido), os máximos de refletância correspondem aos máximos de absorção sumarizados na Tabela 1.
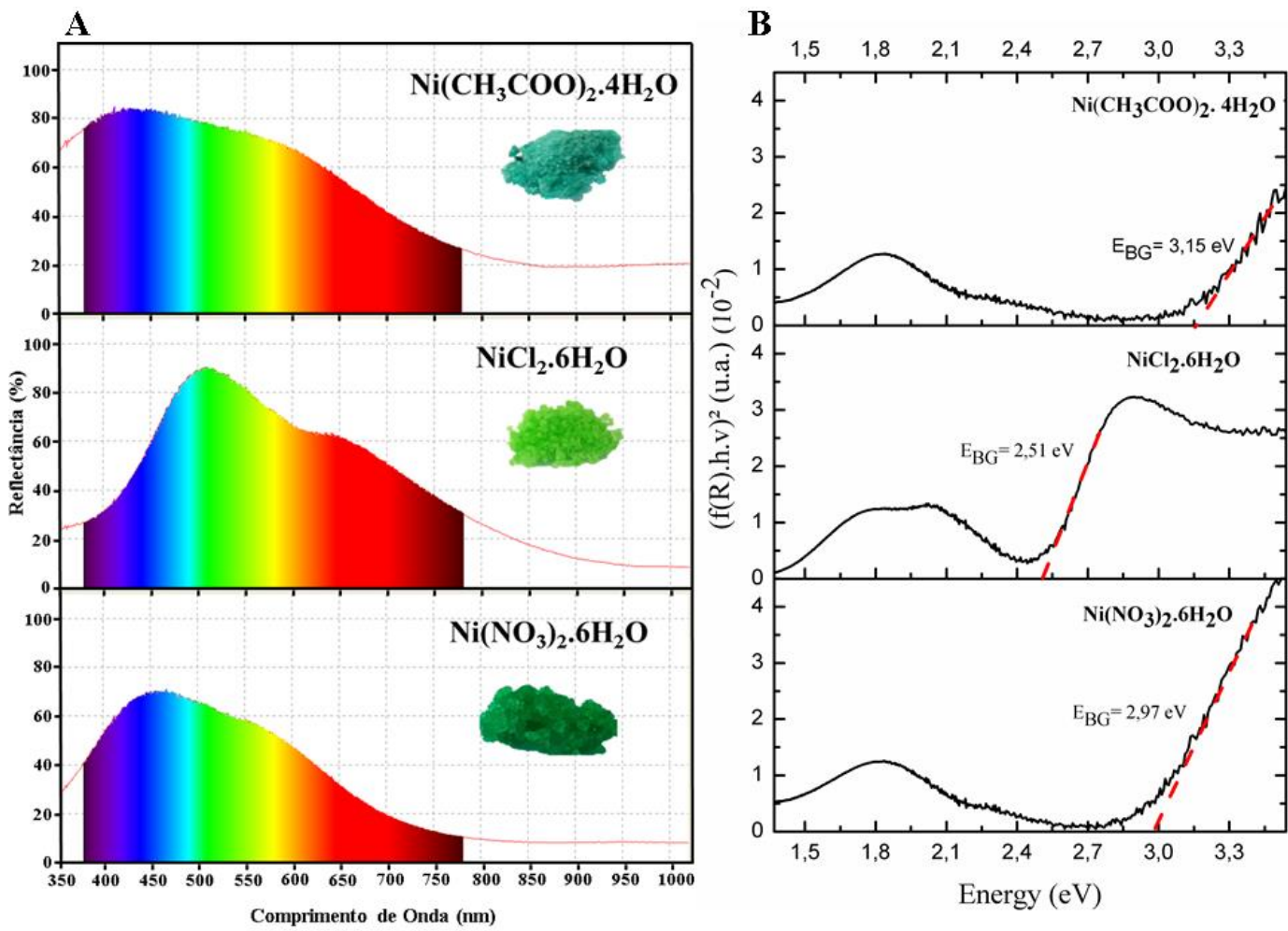

Figura 3: Fotografia digital e espectros eletrônicos (UV-Vis) por refletância difusa dos sais de níquel; e seus respectivos espectros de energia pelo método de WOOD e TAUC [30]. 
$\mathrm{O}$ acetato de níquel apresenta uma banda entre 380 e $450 \mathrm{~nm}\left(\lambda_{\max } \mathrm{em} 430 \mathrm{~nm}\right.$, região espectral do azul) e uma banda larga que inicia em $550 \mathrm{~nm}$ e se estende até $800 \mathrm{~nm}\left(\lambda_{\max }\right.$ em $590 \mathrm{~nm}$, laranja). O cloreto de níquel apresenta uma banda entre 450 e $600 \mathrm{~nm}$ ( $\lambda_{\max }$ em $515 \mathrm{~nm}$, verde) e uma banda entre 600 e $800 \mathrm{~nm}$ $\left(\lambda_{\max }\right.$ em $650 \mathrm{~nm}$, vermelho). Enquanto que, o nitrato de níquel apresenta uma banda entre 400 e $520 \mathrm{~nm}$ com $\lambda_{\max }$ em $450 \mathrm{~nm}$ (azul) e uma banda entre 520 e $620 \mathrm{~nm}$ com $\lambda_{\max }$ em $580 \mathrm{~nm}$ (amarelo).

Percebe-se pela cor apresentada pelos sais, que os perfis de refletância têm diferenças significativas e implicações no comportamento semicondutor dos sais (Figura 3B), ou seja, nos valores da energia de bandgap óptico $\left(\mathrm{E}_{\mathrm{BG}}\right)$. Os espectros de energia (Figura $3 \mathrm{~B}$ ) foram obtidos a partir dos espectros eletrônicos (Figura 3A) usando o método proposto por WOOD e TAUC [30]. Os espectros de energia permitem determinar a energia de "band-gap" $\left(\mathrm{E}_{\mathrm{BG}}\right)$ óptico de sólidos, neste caso dos sais de níquel. Segundo WOOD E TAUC [30]o coeficiente de absorção $\mu$ da energia de band-gap do sólido é obtido diretamente pela Equação 1.

$$
\mu h r=A\left(h v-E_{B G}\right)
$$

Onde: A é uma constante distinta para diferentes transições; $\mathrm{E}_{\mathrm{BG}}$ é a energia de band-gap a ser determinada; hr é a energia do fóton e, n pode assumir valores de 1/2,3/2, 2 e 3 é dependente da natureza de transição eletrônica, que é responsável pela reflexão.

Os valores de band-gap obtidos a partir dos espectros de energia (Figura 3B), em ordem decrescente, foram de: $3,15 \mathrm{eV}$ para acetato; $2,51 \mathrm{eV}$ para nitrato e $2,97 \mathrm{eV}$ para cloreto de níquel. Esses valores estão de acordo com a faixa de energia esperada $(3,18-2,73 \mathrm{eV})$ para a faixa de $390-455 \mathrm{~nm}$ de comprimento de onda [31].

\subsection{Colorimetria - método CIELab}

As medidas de CIELab (Figura 4) foram realizadas para enfatizar a diferença de tons entre sais de níquel na forma sólida que, a olho nu, são facilmente distinguíveis. O método CIELab, leva em consideração o sistema da Comissão Internacional de Iluminação (CIE) em termos de coordenadas $L^{*} a^{*} b^{*}$, onde L considera a luminosidade, a representa o espaço do vermelho ao verde, e b do amarelo ao azul. Os valores calculados de $\mathrm{L} * \mathrm{a} * \mathrm{~b} *$ foram: $77 *-22,2 * 2,6 *$ para acetato; $87,2 *-27,2 * 44,7 *$ para cloreto e $75,7 *-35,6 * 8,1 *$ para nitrato de níquel. A análise desses resultados atribui alta luminosidade (valor $\mathrm{L}^{*}$ ) para todos os sais, sendo maior para cloreto de níquel. Para os sais em solução aquosa vale ressaltar a discussão da Figura 2, onde evidência a formação do íon complexo[Ni( $\left.\left.\mathrm{H}_{2} \mathrm{O}\right)_{6}\right]^{2+}$, hexa(aquo)níquel(II), de coloração verde para todos os sais.

A coordenada a* (avalia o conteúdo de vermelho a verde) resultou em valores negativos para todos os sais, inferindo uma tendência para a coloração verde, sendo que, a menor e a maior tendência referente a essa coordenada, são atribuídas ao acetato e ao nitrato de níquel respectivamente. A coordenada b* (que avalia o conteúdo de amarelo a azul) apresenta valores positivo para todos os sais, evidenciando uma tendência para o amarelo, sendo o maior valor desta coordenada atribuído ao cloreto de níquel. Resumindo podemos dizer que, em termos de tom verde têm-se a seguinte sequência: nitrato > cloreto > acetato.

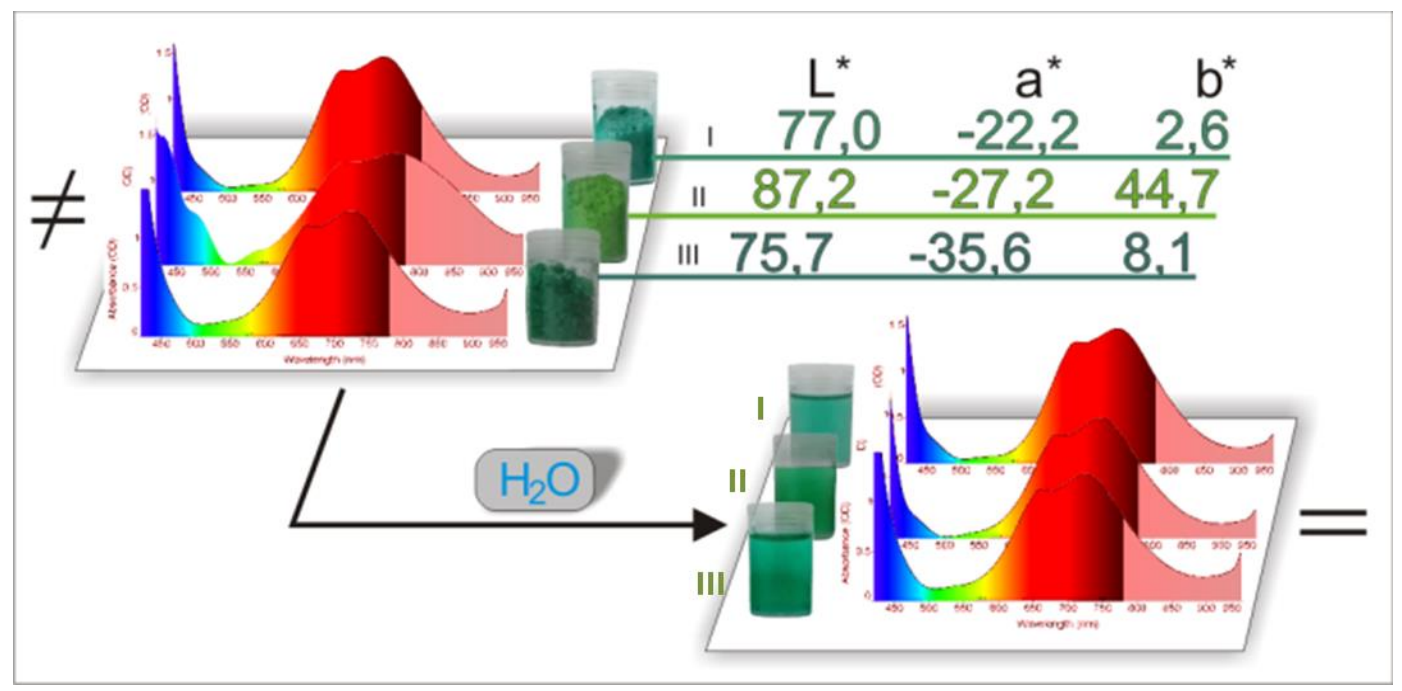

Figura 4: Espectros de absorção (UV-Vis) e medidas colorimétricas ( $\mathrm{L}^{*} \mathrm{a} * \mathrm{~b} *$ ) para sais de níquel na forma sólida e comparativo em solução aquosa: I) $\mathrm{Ni}\left(\mathrm{CH}_{3} \mathrm{COO}\right)_{2} \cdot 4 \mathrm{H}_{2} \mathrm{O}$, II) $\mathrm{NiCl}_{2} \cdot 6 \mathrm{H}_{2} \mathrm{O}$ III $) \mathrm{Ni}\left(\mathrm{NO}_{3}\right) \cdot 6 \mathrm{H}_{2} \mathrm{O}$. 


\subsection{Comportamento Térmico (TG-DTG-DTA)}

A partir de curvas de análise térmica simultânea (TG-DTG-DTA) dos sais de níquel (Figura 5) foi possível determinar a pureza de sais inorgânicos, além da sua composição estequiométrica, quantidade de água estrutural e de hidratação (Tabela 2).
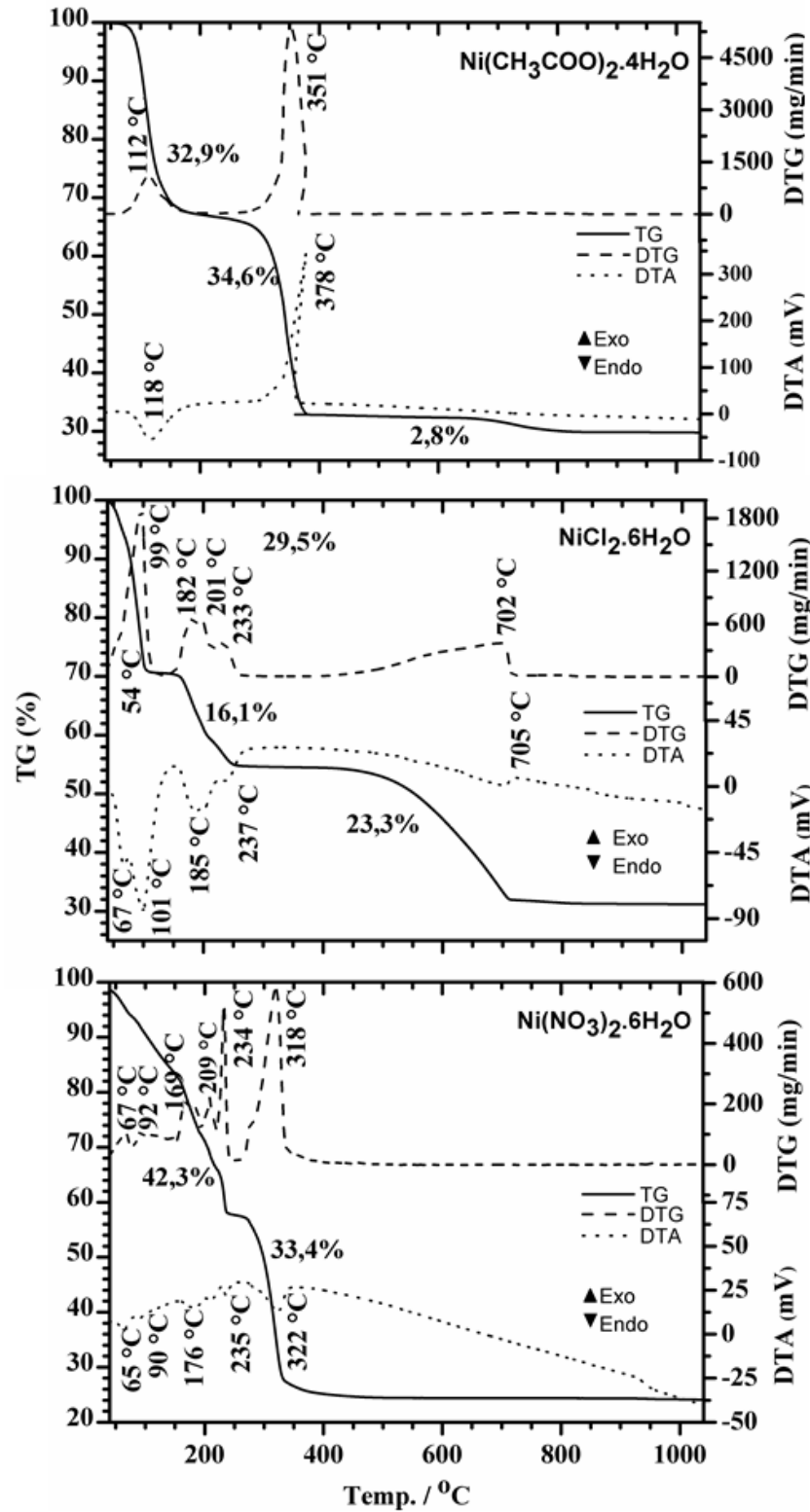

Figura 5: Curvas térmicas (TG-DTG-DTA) obtida dos sais de níquel.

\subsubsection{Decomposição do acetato de níquel tetra-hidratado $\left[\mathrm{Ni}\left(\mathrm{CH}_{3} \mathrm{COO}\right)_{2} \bullet 4 \mathrm{H}_{2} \mathrm{O}\right]$}

Observa-se na Tabela 2, que após a etapa de desidratação do acetato de níquel (32,9\%, pico endotérmico em $118^{\circ} \mathrm{C}$ ), ocorre mais dois eventos de perda de massa de $34,6 \%\left(250\right.$ a $\left.400^{\circ} \mathrm{C}\right)$ com pico exotérmico em 378 ${ }^{\circ} \mathrm{C}$ e outro com $2,8 \%$ perda de massa $\left(650-850^{\circ} \mathrm{C}\right)$ com formação do resíduo $(29,7 \%$ de óxido de níquel). Os dados experimentais da etapa de desidratação permitem determinar a quantidade de moléculas de água presentes no sal acetato de níquel, correspondendo ao valor de 4,81 moléculas. Após a etapa de desidratação, os dados experimentais apontam um valor de 67,1\% de massa residual, divergindo do valor teórico de 71,0\%.

Segundo JESUS et al. [32], esta diferença pode ser um indicativo de que ocorra reações de hidrólise intermediárias na fase gasosa, envolvendo os grupos acetatos durante a desidratação do $\mathrm{Ni}\left(\mathrm{CH}_{3} \mathrm{COO}\right)_{2} \bullet 4 \mathrm{H}_{2} \mathrm{O}$.

A recombinação resultaria na formação simultânea de ácido acético na fase gasosa, gerando acetato básico de níquel na fase sólida. Desta maneira, o primeiro evento de decomposição do acetato de níquel, seguiria a sequência de reações 1, 2, 3 e 4 (Tabela 2). A formação de água em função da decomposição justifi- 
caria a diferença de moléculas de água encontradas experimentalmente $(4,81)$ em relação à quantidade calculada de quatro moléculas.

O segundo evento é caracterizado pela decomposição exotérmica do acetato de níquel, (250a $\left.400{ }^{\circ} \mathrm{C}\right)$, com perda de massa de $34,6 \%$, e o terceiro evento $\left(650\right.$ a $\left.850{ }^{\circ} \mathrm{C}\right)$ é atribuído a perda de massa de $2,8 \%$ referente à decomposição do hidróxido de níquel $\left(\mathrm{Ni}(\mathrm{OH})_{2}\right)$ formado durante o segundo evento. $\mathrm{O}$ resíduo obtido ao fim da análise térmica foi de $29,7 \%$, valor próximo do teórico de 30,0\% para óxido de níquel (NiO). De acordo com JESUS et al. [32], tanto o acetato de níquel quanto o hidróxido de níquel, se decompõem em temperaturas distintas, sendo que ambos geram como resíduo $\mathrm{NiO}$. O acetato de níquel decompõe em temperaturas mais baixas, ao passo que hidróxido de níquel decompõe em temperaturas mais elevadas de acordo com as reações 5 e 6 (Tabela 2). Portanto, a decomposição térmica do acetato de níquel envolve três eventos gerando como resíduo NiO.

Tabela 2: Reações propostas para a desidratação e decomposição dos sais.

\begin{tabular}{|c|c|c|c|c|}
\hline SAL DE NÍQUEL & EVENTO & $\Delta \mathbf{T} /{ }^{\circ} \mathrm{C}$ & DTA $/{ }^{\circ} \mathbf{C}$ & $\begin{array}{l}\text { PERDA DE } \\
\text { MASSA }(\%)\end{array}$ \\
\hline \multirow{6}{*}{$\mathrm{Ni}\left(\mathrm{CH}_{3} \mathrm{COO}\right)_{2}$} & $\mathrm{Ni}\left(\mathrm{CH}_{3} \mathrm{COO}\right)_{2} \cdot 4 \mathrm{H}_{2} \mathrm{O} \rightarrow \mathrm{Ni}\left(\mathrm{CH}_{3} \mathrm{COO}\right)_{2}+4 \mathrm{H}_{2} \mathrm{O}$ & \multirow[t]{2}{*}{$25-250$} & \multirow[t]{2}{*}{118 (endo) } & \multirow[t]{2}{*}{32,9} \\
\hline & $\begin{array}{l}\mathrm{Ni}\left(\mathrm{CH}_{3} \mathrm{COO}\right)_{2} .4 \mathrm{H}_{2} \mathrm{O} \rightarrow 0.86 \mathrm{Ni}\left(\mathrm{CH}_{3} \mathrm{COO}\right)_{2}+ \\
0.14 \mathrm{Ni}(\mathrm{OH})_{2}+0.28 \mathrm{CH}_{3} \mathrm{COOH}+3,72 \mathrm{H}_{2} \mathrm{O}\end{array}$ & & & \\
\hline & $\mathrm{CH}_{3} \mathrm{COOH} \rightarrow \mathrm{CH}_{2} \mathrm{CO}+\mathrm{H}_{2} \mathrm{O}$ & \multirow[t]{3}{*}{$250-400$} & \multirow[t]{3}{*}{378 (exo) } & \multirow[t]{3}{*}{34,6} \\
\hline & $2 \mathrm{CH}_{3} \mathrm{COOH} \rightarrow \mathrm{CH}_{3} \mathrm{CO}+2 \mathrm{CO}_{2}+2 \mathrm{H}_{2} \mathrm{O}$ & & & \\
\hline & $\mathrm{Ni}\left(\mathrm{CH}_{3} \mathrm{COO}\right)_{2} \rightarrow \mathrm{NiO}+\mathrm{CH}_{3} \mathrm{COCH}_{3}+\mathrm{CO}_{2}$ & & & \\
\hline & $\mathrm{Ni}(\mathrm{OH})_{2} \rightarrow \mathrm{NiO}+\mathrm{H}_{2} \mathrm{O}$ & $650-850$ & - & 2,8 \\
\hline \multirow{3}{*}{$\mathrm{NiCl}_{2}$} & $\mathrm{NiCl}_{2} \cdot 6,03 \mathrm{H}_{2} \mathrm{O}_{(\mathrm{s})} \rightarrow \mathrm{NiCl}_{2} \cdot 2,13 \mathrm{H}_{2} \mathrm{O}_{(\mathrm{s})}+3,90 \mathrm{H}_{2} \mathrm{O}$ & \multirow{3}{*}{$\begin{array}{c}30-130 \\
130-375 \\
400-750 \\
\end{array}$} & 206 (endo) & \multirow{3}{*}{$\begin{array}{l}29,5 \\
16,1 \\
23,3 \\
\end{array}$} \\
\hline & $\mathrm{NiCl}_{2} \cdot 2,13 \mathrm{H}_{2} \mathrm{O}_{(\mathrm{s})} \rightarrow \mathrm{NiCl}_{2(\mathrm{~s})}+2,13 \mathrm{H}_{2} \mathrm{O}$ & & 237 (endo) & \\
\hline & $\mathrm{NiCl}_{2(\mathrm{~s})}+\mathrm{O}_{2} \rightarrow \mathrm{NiO}_{(\mathrm{s})}+\mathrm{Cl}_{2(\mathrm{~g})}$ & & 705 (endo) & \\
\hline \multirow{2}{*}{$\mathrm{Ni}\left(\mathrm{NO}_{3}\right)_{2}$} & $\mathrm{Ni}\left(\mathrm{NO}_{3}\right)_{2} \cdot 6,89 \mathrm{H}_{2} \mathrm{O}_{(\mathrm{s})} \rightarrow \mathrm{Ni}\left(\mathrm{NO}_{3}\right)_{2(\mathrm{~s})}+6,89 \mathrm{H}_{2} \mathrm{O}$ & \multirow{2}{*}{$\begin{array}{l}30-260 \\
260400\end{array}$} & 235 (endo) & \multirow{2}{*}{$\begin{array}{l}42,3 \\
33,4 \\
\end{array}$} \\
\hline & $\mathrm{Ni}\left(\mathrm{NO}_{3}\right)_{2(\mathrm{~s})} \rightarrow \mathrm{NiO}_{(\mathrm{s})}+2 \mathrm{NO}_{2(\mathrm{~g})}+1 / 2 \mathrm{O}_{2(\mathrm{~g})}$ & & 316 (endo) & \\
\hline
\end{tabular}

\subsubsection{Decomposição do cloreto de níquel hexa-hidratado $\left[\mathrm{NiCl}_{2} \cdot 6 \mathrm{H}_{2} \mathrm{O}\right]$}

Os dados referentes ao cloreto de níquel (Tabela 2) destacam de forma geral a presença de três eventos de perda de massa. Onde, os dois primeiros correspondem a perdas de moléculas de água envolvendo processos endotérmicos. Ao longo desses dois eventos a temperatura aumenta gradualmente de 30 a $375{ }^{\circ} \mathrm{C}$, o que implica na saída inicial de moléculas de água fracamente ligadas (superficial), seguida pela saída de água fortemente retida na estrutura do sal.

Para a decomposição da massa inicial de $19,33 \mathrm{mg}$ de cloreto de níquel determinou-se experimentalmente a quantidade de 6,03 moléculas de água, muito próximo do valor teórico que é de 6 moléculas de água de hidratação. Considerando esta composição e os valores de perda de massa envolvida em cada evento, foi possível propor reações de desidratação e decomposição do cloreto de níquel, que resulta em $31,1 \%$ (6,22 mg) de resíduo final (Tabela 2).

No primeiro evento $\left(29,5 \%\right.$ de perda de massa entre $\left.30-130{ }^{\circ} \mathrm{C}\right)$, uma pequena fração de moléculas de água de cristalização sofre fusão a baixas temperaturas, enquanto que, no segundo evento $(16,1 \%$ de perda de massa) ocorre maior perda de água (entre 130 e $375^{\circ} \mathrm{C}$ ) referente à desidratação, saída de moléculas de água mais fortemente ligadas. $\mathrm{O}$ terceiro evento, caracterizado também como um processo endotérmico, corresponde a decomposição térmica do cloreto de níquel a óxido de níquel (NiO), com possível liberação de gás cloro $\left(\mathrm{Cl}_{2}\right)$.

\subsubsection{Decomposição do nitrato de níquel hexa-hidratado $\left[\mathrm{Ni}\left(\mathrm{NO}_{3}\right)_{2} \cdot 6 \mathrm{H}_{2} \mathrm{O}\right]$}

As curvas térmicas para o nitrato de níquel hexa-hidratado mostram que a decomposição envolve duas perdas de massa principais. A primeira (entre 30 e $260{ }^{\circ} \mathrm{C}$ ) é atribuída ao processo de desidratação do sal; a segunda perda de massa (entre 260 e $1000{ }^{\circ} \mathrm{C}$ ) atribui-se à formação do óxido de níquel (NiO). 
Os resultados obtidos a partir da derivada da curva TG (DTG) mostram de forma mais detalhada a decomposição térmica do $\mathrm{Ni}\left(\mathrm{NO}_{3}\right)_{2}{ }^{\circ} 6 \mathrm{H}_{2} \mathrm{O}$. Até $220{ }^{\circ} \mathrm{C}$ observam-se três eventos endotérmicos, referentes a saídas de água fracamente ligadas à estrutura, seguida pela saída de água estrutural fortemente ligada (pico em $\left.235^{\circ} \mathrm{C}\right)$. O evento endotérmico acima de $260^{\circ}$ Crefere-se à perda de massa $(33,4 \%)$ para formação de $\mathrm{NiO}$ com possível saída de dióxido de nitrogênio e oxigênio (Tabela 2).

\subsection{Análise morfológica (MEV-EDS) e estrutural (DRX) dos resíduos da análise térmica(TG)}

Os resíduos das curvas térmicas são propostos (Tabela 3) como sendo óxidos de níquel (NiO). Com intuito de averiguar essa proposição foram obtidas imagens de microscopia eletrônica (MEV) com respectivos espectros de energia dispersiva (EDS) para os resíduos finais de cada sal de níquel.

A Figura 6A corresponde ao $\mathrm{NiO}$ obtido a partir do acetato de níquel, apresenta-se na forma de um "puff", onde as partículas permanecem agregadas entre si, porém não uniformes. Por outro lado, não se observa diferença de morfologia quando se compara os resíduos obtidos da queima do cloreto (Figura 6B) e do nitrato (Figura 6C). Os agregados formados em cada material são significativamente parecidos, porém o tamanho é da ordem de $0,2 \mu \mathrm{m}$ para acetato e cloreto (Figura 6A e B) e da ordem de $0,1 \mu \mathrm{m}$ para nitrato (Figura 6C).

Os dados analíticos (Tabela 3) foram obtidos dos gráficos de EDS, que confirmam a formação de oxido de níquel como produto final de todos os sais. Porém, a composição estimada varia em frações que podem estar relacionadas com a natureza dos cátions (acetato, cloreto e nitrato) presentes no produto final e de difícil mensuração por EDS. Também pode estar relacionado com o ambiente térmico (rampa de aquecimento, vazão do ar comprimido, tipo de porta amostra utilizado). Destaca-se que o resíduo do nitrato de níquel $\left(\mathrm{NiO}_{1,1}\right)$ apresenta a composição mais próxima do teórico $(\mathrm{NiO})$.

Tabela 3: Dados analíticos (EDS) e composição estimada do resíduo de TG.

\begin{tabular}{c|c|c|c|c}
\hline AMOSTRA & $\mathbf{N i}(\boldsymbol{\%})$ & $\mathbf{O}(\boldsymbol{\%})$ & RAZÃO (Ni/O) & $\begin{array}{c}\text { COMPOSIÇÃO } \\
\text { ESTIMADA }\end{array}$ \\
\hline Teórico & 78,58 & 21,42 & 3,67 & $\mathrm{NiO}-$ óxido de níquel \\
\hline Acetato & 74,07 & 25,93 & 2,85 & $\mathrm{NiO}_{1,3}$ \\
\hline Cloreto & 86,29 & 13,71 & 6,29 & $\mathrm{NiO}_{0,6}$ \\
\hline Nitrato & 77,06 & 22,94 & 3,35 & $\mathrm{NiO}_{1,1}$ \\
\hline
\end{tabular}

Os difratogramas de raios X (Figura 6) para os óxidos de níquel obtidos a partir da calcinação dos sais apresentam perfil de sólidos policristalinos com três picos característicos (entre $30^{\circ} \mathrm{e} 70^{\circ} 2 \theta$ ), indexados (hkl) como: (111), (200) e (220) de acordo com a carta cristalográfica: PDF 01-073-1519. Ao analisar os dados sumarizados na Tabela 4, destaca-se que, todas as amostras foram identificadas como óxido de níquel (NiO), porém ocorre uma variação na posição e intensidade dos picos, atribuído como diferença imposta pelo sal precursor.

Avaliando em relação ao pico principal (200, intensidade 100\%) o tamanho de cristalito diminui do $\mathrm{NiO}$-acetato $(447,9 \mu \mathrm{m})>\mathrm{NiO}$-cloreto $(408,5 \mu \mathrm{m})>\mathrm{NiO}$-nitrato $(321,8 \mu \mathrm{m})$, enquanto que o grau de cristalinidade aumenta NiO-cloreto $(85,7 \%)<\mathrm{NiO}$-acetato $(95,6 \%)<\mathrm{NiO}$-nitrato $(95,8 \%)$, sugerindo que na busca por menor tamanho de partícula com maior cristalinidade deve-se fazer uso do precursor nitrato de níquel hexahidratado.

Tabela 4: Dados de difração de raio X: posições em ângulo $2 \theta$ e valores de distância (d) para picos indexados (hkl), tamanho () e grau de cristalinidade (\%) para pico principal (200).

\begin{tabular}{|c|c|c|c|c|c|c|}
\hline \multirow{2}{*}{ AMOSTRA } & \multirow{2}{*}{ PARÂMETROS } & \multicolumn{3}{|c|}{ INDEXAÇÕES (hkl) } & \multirow{2}{*}{$\begin{array}{l}\text { TAMANHO DE- } \\
\text { CRISTALITO }(\mu \mathrm{m})\end{array}$} & \multirow{2}{*}{$\begin{array}{l}\text { CRISTALINIDADE } \\
(\%)\end{array}$} \\
\hline & & (111) & (200) & (220) & & \\
\hline \multirow{2}{*}{$\mathrm{NiO}$-acetato } & Posição 20 & 37,17 & 43,21 & 62,81 & \multirow{2}{*}{447,9} & \multirow{2}{*}{95,6} \\
\hline & d $(\AA)$ & 2,416 & 2,092 & 1,478 & & \\
\hline \multirow{2}{*}{ NiO-cloreto } & Posição $2 \theta$ & 36,52 & 42,59 & 62,26 & \multirow{2}{*}{408,5} & \multirow{2}{*}{85,7} \\
\hline & d $(\AA)$ & 2,458 & 2,120 & 1,490 & & \\
\hline \multirow{2}{*}{ NiO-nitrato } & Posição $2 \theta$ & 37,01 & 43,02 & 62,66 & \multirow{2}{*}{321,8} & \multirow{2}{*}{95,8} \\
\hline & d (̊) & 2,427 & 2,100 & 1,481 & & \\
\hline
\end{tabular}



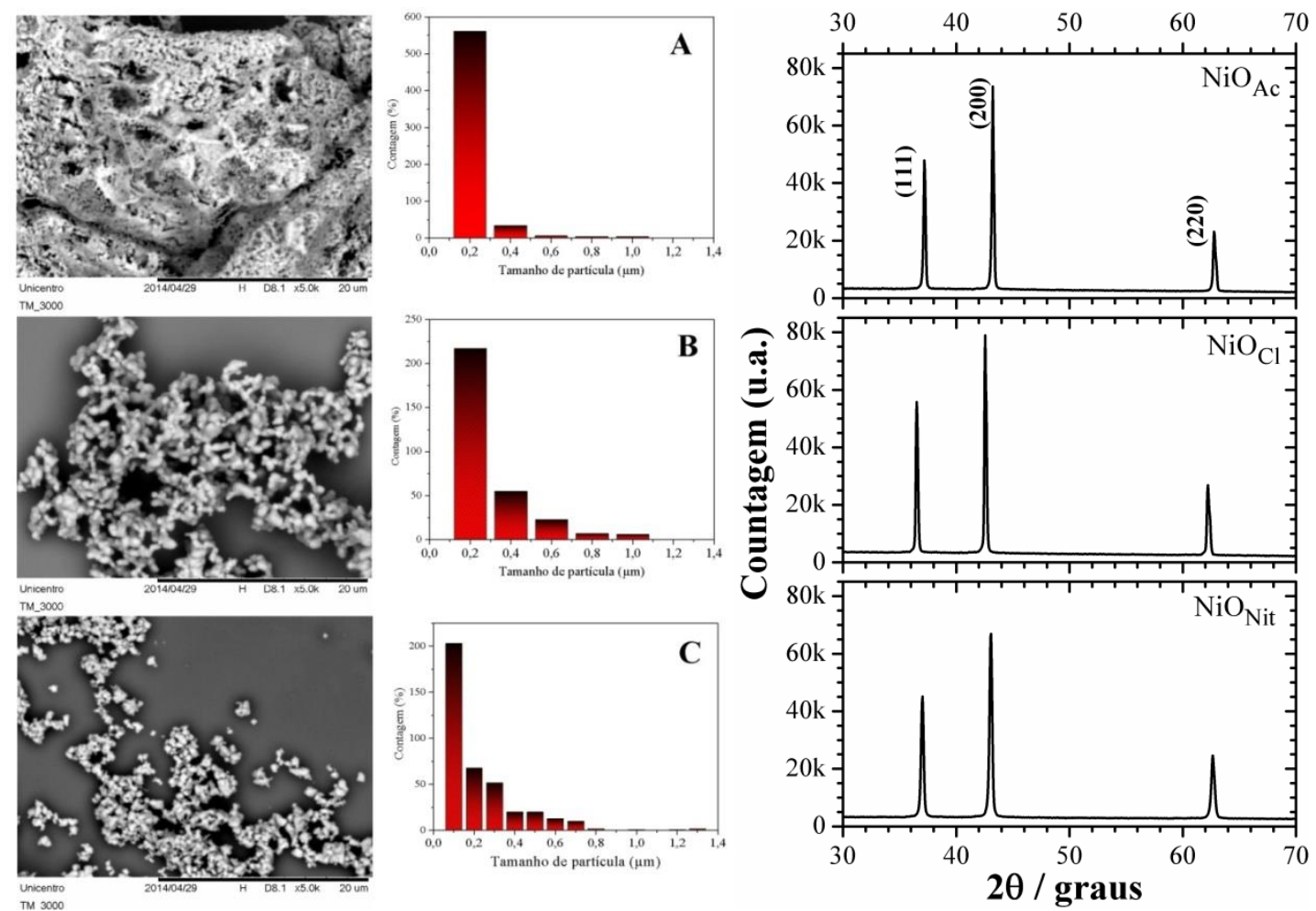

Figura 6: Imagem de MEV, histogramas de tamanho de partícula e difratogramas de raio $\mathrm{X}$ dos resíduos (NiO) obtidos na análise térmica dos sais de níquel: (A) acetato $-\mathrm{NiO}_{\mathrm{Ac}}$, (B) cloreto $-\mathrm{NiO}_{\mathrm{Cl}}$, e (C) nitrato $-\mathrm{NiO}_{\mathrm{Nit}}$.

\section{CONCLUSÕES}

Avaliação de sais inorgânicos se torna importante do ponto de vista analítico, espectroscópico, estrutural, térmico e morfológico para compreensão das técnicas de caracterização e propósitos de comparação com os derivados preparados a partir destes, como óxidos, hidróxidos e oxi-hidróxidos. Difratometria de raios X possibilitou identificar a rede cristalina e correlacionar os parâmetros de rede para indexar os picos de difração característicos de cada sal utilizado.

Os espectros eletrônicos em solução (absorbância) e sólidos (refletância) possibilitaram identificar as transições eletrônicas envolvidas, embora sejam as mesmas, ocorrem em regiões de comprimento de onda distintos. Dados de L*a*b* proporcionam concluir que a intensidade da tonalidade verde diminui na sequência: nitrato > cloreto > acetato.

As curvas de analise térmica possibilitaram determinar a quantidade de moléculas de água de hidratação e sua compatibilidade conforme previsto pelo fornecedor. Possibilitou ainda, a partir do resíduo final, gerar informações morfológicas e estruturais, como tamanho de partículas e identificação da fase formada, com estimativa de composição para óxido de níquel $(\mathrm{NiO})$.

\section{AGRADECIMENTOS}

Os autores agradecem as agências de fomento CNPq (447902/2014-8 e 305447/2013-0), Capes (028/2009 e 063/2010), Finep (01.09.0393.00 e 01.12.0471.00).

\section{BIBLIOGRAFIA}

[1] ZARBIN, A. J. G., “Química de (nano)materiais”, Química Nova, v. 30, n. 6, pp. 1469-1479, 2007.

[2] SCHAUMLÖFFEL, D. J. "Nickel species: analysis and toxic effects", Journal of Trace Elements in Medicine and Biology, v. 26, pp. 1-6, 2012.

[3] ISMAIL, R. A., GHAFORI, S., KADHIM, G. A. "Preparation and characterization of nanostructured nickel oxide, thin films by spray pyrolysis", Applied Nanoscience, v.3, pp. 509-514, 2013

[4] CHENG, M. Y., HWANG, B. J., "Transformation of $\beta-\mathrm{Ni}(\mathrm{OH})_{2}$ to $\mathrm{NiO}$ nano-sheets via surface nanocrystalline zirconia coating: Shape and size retention", Nanoscale Research Letters, v.2, pp. 2-28, 2006 
[5] ERTAŞ, F. S., KAŞ, R., ÜNAL, U., et al., "Sonochemical synthesis and electrochemical characterization of $\alpha$-nickel hydroxide: precursor effects", Journal Solid State Electrochemistry, v. 17, n. 5, pp. 1455-1462, 2013.

[6] DANCZUK, M., NUNES Jr., C. V., ARAKI, K., et al., "Influence of alkaline cation on the electrochemical behavior of stabilized alpha-Ni(OH $)_{2}$ ", Journal Solid State Electrochemistry, v. 18, pp. 2279-2287, 2014.

[7] ROCHA, M. A., WINNISCHOFER, H., ARAKI, K., et al., "A New Insight on the Preparation of Stabilized Alpha-Nickel Hydroxide Nanoparticles", Journal of Nanoscience and Nanotechnology, v. 11, n. 5, pp. 3985-3996, 2011.

[8] GIAROLA, D. A., SILVA, P. R. C., URBANO, A., et al., "Surfactant effect on electrochemical-induced synthesis of $\alpha-\mathrm{Ni}(\mathrm{OH})_{2}$ ", Journal Solid State Electrochemistry, v. 18, n. 2, pp. 497-504, 2014.

[9] BODE, H., DELMELT, K., WITTE, J.,"Zurkenntnis der nickel hydroxide electrode I. Über das nickel (II)-hydroxidhydrat", Electrochimica Acta, v. 11, n. 8, pp. 1079-1087, 1966.

[10] TOWER, O. F., "Note on Colloidal Nickel Hydroxide", The Journal of Physical Chemistry, v. 28, n. 2, pp. 176-178, 1924.

[11] SAKAI, G., MIYAZAKI, M., KIJIMA, T., "Synthesis of $\beta$ - $\mathrm{Ni}(\mathrm{OH})_{2}$ Hexagonal Plates and Electrochemical Behavior as a Positive Electrode Material", Journal of The Electrochemical Society, v. 157, n. 8, pp. A932-A939, 2010.

[12] KIANI, M. A., MOUSAVIS, M. F., GHASEMI, S., "Size effect investigation on battery performance: Comparison between micro-as nickel battery cathode material and nano-particles of $\beta-\mathrm{Ni}(\mathrm{OH})_{2}$ ", Journal of Power Sources, v. 195, n. 17, pp. 5794-5800, 2010.

[13] ZHANG, W., JIANG, W., YU, L., et al., "Effect of nickel hydroxide composition on the electrochemical performance of spherical $\mathrm{Ni}(\mathrm{OH})_{2}$ positive materials for $\mathrm{Ni}-\mathrm{MH}$ batteries", International Journal of Hydrogen Energy, v. 34, n. 1, pp. 473-480, 2009.

[14] MARTinS, P.R., ANGNES, L., ROCHA, M. A., et al., "Highly Sensitive Amperometric Glucose Sensors Based on Nanostructured $\alpha-\mathrm{Ni}(\mathrm{OH}) 2$ Electrodes", Electroanalysis, v. 23, n. 11, pp. 2541-2548, 2011.

[15] CISZEWSKI, A.; STEPNIAK, I., "Nanoparticles of $\mathrm{Ni}(\mathrm{OH})_{2}$ embedded in chitosan membrane as electrocatalyst for non-enzymatic oxidation of glucose", Electrochimica Acta, v. 111, pp. 185-191, 2013.

[16] MARTINS, P. R., FERREIRA, L. M. C., ARAKI, K., et al., "Influence of cobalt content on nanostructured alpha-phase-nickel hydroxide modified electrodes for electrocatalytic oxidation of isoniazid", Sensors and Actuators. B: Chemical, v. 192, pp. 601-606, 2014.

[17] MODOLO, M. L., DANCZUK, M., ANAISSI, F. J., et al., "Carbon Ceramic Electrodes Modified with Alpha-Nickel Hydroxide Applied to the Electro-Oxidation of Methanol in Alkaline Medium", ECS Transactions, v. 61, n. 1, pp. 319-330, 2014.

[18] YAN, W., WANG, D., DIAZ, L., et al., "Nickel nanowires as effective catalysts for urea electrooxidation", Electrochimica Acta, v. 134, pp. 266-271, 2014.

[19] INAMDAR, A. I., SONAVANE, C. A., PAWAR, S. M., et al., "Electrochromic and electrochemical properties of amorphous porous nickel hydroxide thin films”, Applied Surface Science, v. 257, pp. 9606-9611, 2011.

[20] HU, C., CHEN, J.-C., CHANG, K.-H. "Cathodic deposition of $\mathrm{Ni}(\mathrm{OH})_{2}$ and $\mathrm{Co}(\mathrm{OH})_{2}$ for asymmetric supercapacitors: Importance of the electrochemical reversibility of redox couples”, Journal of Power Sources, v. 221, pp. 128-133, 2013.

[21] NUNES JUNIOR, C. V., DANCZUK, M., BORTOTI, A. A., et al., "Unexpected effect of drying method on the microstructure and electrocatalytic properties of bentonite/alpha-nickel hydroxide nanocomposite", Journal of Power Sources, n. 297, p. 408-412, 2015.

[22] TOMA, H. E., BONIFÁCIO, L. S., ANAISSI, F. J., "Da cor à cor inexistente: uma reflexão sobre espectros eletrônicos e efeitos cromáticos”, Química Nova, v. 28, n. 5, pp. 897-900, 2005.

[23] GUSHIKEM, Y., "Espectros Eletrônicos de Alguns Complexos de Geometria Octaédrica de $\mathrm{Ni}^{2+}: \mathrm{Uma}^{2}$ Introdução Prática à Teoria do Campo Cristalino no Curso de Graduação", Química Nova, v. 28, n. 1, pp. 153-156, 2005.

[24] LEAL, B. C., LUZA, L., BAIBICH, I., et al., “Abordagem Teórico-Experimental da Teoria do Campo Cristalino na disciplina de Síntese inorgânica”, Química Nova, v. 34, n. 10, pp. 1830-1835, 2011. 
[25] COTTON, F. A., WILKINSON, G., MURILlO, C. A., et al., Advanced Inorganic Chemistry, 6th ed.; New York, Wiley, 1999.

[26] TOMA, H. E., "Teoria do Campo Ligante”, In: Toma, H. E., Química de Coordenação, Organometálica e Catálise, Blucher: 1 ed., Capitulo 4, São Paulo, Blucher, 2013.

[27] Li, W.L., ZHOU, G.D., WAK, T.C.W. Advanced Structural Inorganic Chemistry, Oxford University Press, 2008 .

[28] LOW, W. "Paramagnetic and Optical Spectra of Divalent Nickel in Cubic Crystalline Fields", Physical Review, v. 109, pp. 247-255, 1958.

[29] YANG, F., TU, C. "Growth and Spectroscopy of $\mathrm{Ni}^{2+}$ in $\mathrm{ZnWO}_{4}$ Crystal", Materials Letters, v.61, pp.3056-3058, 2007.

[30] WOOD, D. L., TAUC, J. "Weak Absorption tails in Amorphous Semiconductors", Physical Review B, v.5, n.8, pp. 3144-3151, 1972.

[31] SOLÉ, J. G., BAUSÁ, L. E., JAQUE, D. AnIntroduction to the Optical Spectroscopy of Inorganic Solids, $1^{\text {a }}$ ed., Wiley, 2005.

[32] JESUS, J. C., GONZÁLEZ, I., QUEVEDO, A., et al., "Thermal Decomposition of Nickel Acetate Tetrahydratean Integrated Study by TGA, QMS and XPS Techniques", Journal of Molecular Catalysis A: Chemical, v. 228, pp. 283-291, 2005. 